\title{
Duplication of an upstream silencer of FZP significantly increases grain yield in rice (Abstract)
}

\author{
Yongzhong Xing \\ National Key Lab of Crop Genetic improvement, Huahzong Agricultural University, 430070, Wuhan China \\ Corresponding author: yzxing@ mail.hzau.edu.cn
}

\begin{abstract}
Transcriptional silencer and copy number variants (CNVs) are associated with gene expression. However, their roles in generating phenotypes have not been well studied. In this study, we identified a rice quantitative trait locus (QTL), SGDP7 (Small Grain and Dense Panicle 7). SGDP7 is identical to FZP (FRIZZLE PANICLE), which represses the formation of axillary meristems. An 18-bp fragment, named CNV-18bp, was inserted $\sim 5.3$ $\mathrm{kb}$ upstream of FZP, thus resulting in a tandem duplication in Chuan 7. The CNV-18bp duplication repressed FZP expression, prolonged the panicle branching period and increased grain yield by more than $15 \%$ by substantially increasing the number of spikelets per panicle (SPP) and slightly decreasing 1000-grain weight (TGW). The transcription repressor OsBZR1 binds the CGTG motifs in CNV-18bp and represses FZP expression, indicating that CNV-18bp is the upstream silencer of FZP. These findings showed that CNVs of the silencer coordinate a trade-off between SPP and TGW by fine-tuning FZP expression, and balancing the tradeoff would enhance yield potential.
\end{abstract}

Keywords: China, rice grain yield, rice QTL, FZP, SGDP7, tandem duplication, transcription repressor, upstream silencer. 
